\title{
Blocking autocrine VEGF signaling by sunitinib, an anti-cancer drug, promotes embryonic stem cell self- renewal and somatic cell reprogramming
}

\author{
Guofang Chen ${ }^{1, *}$, Xinxiu $\mathrm{Xu}^{1,2,{ }^{*} \text {, Lihong Zhang }}{ }^{1}$, Yanbin $\mathrm{Fu}^{2}$, Min Wang ${ }^{1}$, Haifeng $\mathrm{Gu}^{1}$, Xin Xie ${ }^{1,2}$ \\ ${ }^{1}$ CAS Key Laboratory of Receptor Research, the National Center for Drug Screening, Shanghai Institute of Materia Medica, Chi- \\ nese Academy of Sciences, 189 Guo Shou Jing Road, Shanghai 201203, China; ${ }^{2}$ Shanghai Key Laboratory of Signaling and Dis- \\ ease Research, Laboratory of Receptor-based Bio-medicine, School of Life Sciences and Technology, Tongji University, Shanghai \\ 200092, China
}

Maintaining the self-renewal of embryonic stem cells (ESCs) could be achieved by activating the extrinsic signaling, i.e., the use of leukemia inhibitory factor (LIF), or blocking the intrinsic differentiation pathways, i.e., the use of GSK3 and MEK inhibitors (2i). Here we found that even in medium supplemented with LIF, mESCs still tend to differentiate toward meso-endoderm lineages after long-term culture and the culture spontaneously secretes vascular endothelial growth factors (VEGFs). Blocking VEGF signaling with sunitinib, an anti-cancer drug and a receptor tyrosine kinase (RTK) inhibitor mainly targeting VEGF receptors (VEGFRs), is capable of maintaining the mESCs in the undifferentiated state without the need for feeder cells or LIF. Sunitinib facilitates the derivation of mESCs from blastocysts, and the mESCs maintained in sunitinib-containing medium remain pluripotent and are able to contribute to chimeric mice. Sunitinib also promotes iPSC generation from MEFs with only Oct4. Knocking down VEGFR2 or blocking it with neutralizing antibody mimicks the effect of sunitinib, indicating that blocking VEGF/VEGFR signaling is indeed beneficial to the self-renewal of mESCs. We also found that hypoxia-inducible factor alpha (HIF1 $\alpha)$ and endoplasmic reticulum (ER) stress are involved in the production of VEGF in mESCs. Blocking both pathways inhibits the expression of VEGF and prevents spontaneous differentiation of mESCs. Interestingly, LIF may also exert its effect by downregulating HIF1 $\alpha$ and ER stress pathways and subsequent VEGF expression. These results indicate the existence of an intrinsic differentiation pathway in mESCs by activating the autocrine VEGF signaling. Blocking VEGF signaling with sunitinib or other small molecules help to maintain the mESCs in the ground state of pluripotency.

Keywords: sunitinib; Sutent; embryonic stem cell; self-renewal; iPSC; VEGF; autocrine

Cell Research (2014) 24:1121-1136. doi:10.1038/cr.2014.112; published online 22 August 2014

\section{Introduction}

The two essential properties of embryonic stem cells (ESCs), self-renewal and pluripotency, are regulated by a network of signaling from intrinsic factors and extrinsic stimulants such as cytokines and growth factors. Vari-

\footnotetext{
*These two authors contributed equally to this work. Correspondence: Xin Xie

Tel: +86-21-50801313 ex 156

E-mail: xxie@simm.ac.cn

Received 2 April 2014; revised 16 May 2014; accepted 2 June 2014; published online 22 August 2014
}

ous culture conditions, including the use of feeder cells, conditioned media, growth factors, and serum have been used to derive and maintain ESCs [1]. Leukemia inhibitory factor (LIF) is among the earliest cytokines discovered to be able to promote self-renewal of mESCs [2]. LIF binding leads to the dimerization of gp130 and LIF receptor and the activation of Jak/Stat3 signaling pathway. In combination with serum or bone morphogenetic proteins (BMPs), which induce the expression of proteins that suppress differentiation [3], LIF is able to maintain the self-renewal of mESCs. However, these cytokines fail to block the activation of ERK, a known pathway involved in the differentiation of mESCs [4]. This leads 
to the discovery that the combination of small-molecule inhibitors of ERK and GSK3 pathways is able to bypass the need of cytokine signaling and promote the self-replication of mESCs [5]. PD0325901 (MEK inhibitor) and CHIR99021 (GSK3 inhibitor), the so-called 2i, are now commonly used for culturing mESCs and induced pluripotent stem cells (iPSCs). In addition, other MEK or GSK3 inhibitors, such as pluripotin and 6-bromoindirubin-3'-oxime (BIO) [6], and PKC inhibitors [7] have also been reported to promote the self-renewal of mESCs. However, the involvement of other signaling pathways in maintaining the balance between stemness and differentiation remains illusive and the demands for better and easier culture conditions remain high.

Vascular endothelial growth factors (VEGFs) are potent mediators of vasculogenesis in the fetus and angiogenesis in the adults. In mammals, five VEGF ligands have been identified so far. These ligands bind in an overlapping pattern to three receptor tyrosine kinases (RTKs), designated VEGF receptor-1, -2 and -3 (VEGFR1-3), and to co-receptors [8]. In mouse, at least three isoforms of VEGF (VEGFA, VEGFB and VEGFC) have been found and displayed different affinities for the known VEGF receptors and co-receptors, suggesting different roles of these VEGF isoforms [9].

Many early studies have shown that the VEGF and VEGFR family play critical roles in cardiovascular, haematopoietic and lymphatic development. Inactivation of a single VEGFA allele [10] or deletion of VEGFR2 in mice [11] results in early lethality at embryonic stage due to deficient endothelial cell development and lack of vessels. In contrast, overexpression of VEGFA results in aberrant heart development, which also leads to lethality at E12.5-14 [12]. Haematopoietic and endothelial cells have been proposed to share a common early progenitor that expresses VEGF signaling system. Indeed, the survival [13] and migration [14] of haematopoietic stem cells are promoted by VEGF and VEGFRs. In vitro, VEGF has been reported to induce endothelium and cardiac myocyte differentiation from ESCs or iPSCs [15, 16]. VEGF-mediated angiogenesis is activated during tumor formation [17]. This leads to the development of anti-VEGF antibodies and small-molecule RTK inhibitors that target VEGFRs for tumor therapy. Anti-angiogenic drugs targeting the VEGF signaling pathways, including bevacizumab, sorafenib, sunitinib, and have been approved by FDA for the treatment of advanced carcinoma.

In this study, we sought to dissect the roles of autocrine VEGF/VEGFR signaling in the fate determination of mESCs and the generation of iPSCs and discuss the possible application of VEGFR inhibitor sunitinib in stem cell culture.

\section{Results}

mESCs tend to differentiate toward meso-endoderm lineages and produce VEGF during long-term culture

It is generally adopted that ESCs have to be cultured in special medium and passaged every 2-3 days to help maintain the pluripotency of the cells $[3,18]$. Without passage, E14 cells cultured in mES medium for a long period tend to spontaneously differentiate even in the presence of LIF and with daily medium change (Figure 1A). On day 5, E14 cells started to lose the typical compact colony morphology of mESCs (Figure 1A). Similar phenomenon was also observed in E14 cells cultured in N2B27 medium supplemented with LIF or mES medium without LIF (Supplementary information, Figure S1A). The spontaneous differentiation was more obvious and occurred earlier (day 3) in mES medium without LIF (Supplementary information, Figure S1A). Quantitative PCR also confirmed the downregulation of pluripotency genes Oct4, Sox2, Nanog and Rexl (Figure 1B). Among the lineage-specific genes, the ectoderm markers Nestin and Sox 1 were not significantly altered during the spontaneous differentiation, while the mesoderm markers Brachyury and SMA and the endoderm markers GATA4 and Sox 17 were all significantly increased (6.7-, 43.8, 19- and 6.7-fold increase on day 8 relative to those on day 1, respectively; Figure 1C). The downregulation of epithelial cell markers E-cadherin, EP-CAM, Ocln and $C l d n 3$ and the upregulation of mesenchymal cell markers $N$-cadherin, Snail, Slug and Fn indicated that the epithelial-mesenchymal transition (EMT) occurs during longterm culture of mESCs (Figure 1D).

The above data suggested that mESCs tend to differentiate toward meso-endoderm lineages spontaneously during long-term culture. Therefore, we investigated additional genes and found that several genes involved in the vascular differentiation in mesoderm, including $V E G F, V E$-cadherin and PECAM, were all upregulated (Figure 1E). mRNA of $V E G F A$ increased more than that of $V E G F B$ (Figure 1F) after long-term culture and VEGFA protein secreted into the mES media (with LIF) also increased significantly (Figure 1G). Similar increase of $V E G F$ expression was also observed in mESCs cultured in the serum-free N2B27 media (with LIF; Supplementary information, Figure S1B). The expression levels of a panel of growth factors were analyzed in E14 cells cultured in mES media (with LIF) for 1-8 days (Supplementary information, Figure S1C). Many growth factors were not detectable. A couple of growth factors, such as FGF4 and PDGFA, did not show time-dependent change, even though their expression levels were relatively high. $V E G F A$ expressed at a relatively high level and displayed 
most striking upregulation ( $\sim 6$-fold) after 5 days of culture. Another growth factor, $P D G F B$, was also upregulated ( $\sim 3$-fold) in long-term culture, although its expression level was relatively low.

Addition of the recombinant protein $\mathrm{mVEGF}_{164}$ or
PDGFBB induced loss of mESC morphology on day 3, 2 days earlier than the spontaneous differentiation (Figure $1 \mathrm{H}$ and Supplementary information, Figure S2A), indicating that activation of both pathways leads to differentiation. However, knockdown of $V E G F A$ but not $P D G F B$
A
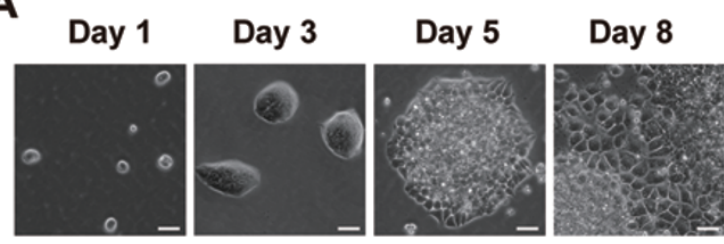

B
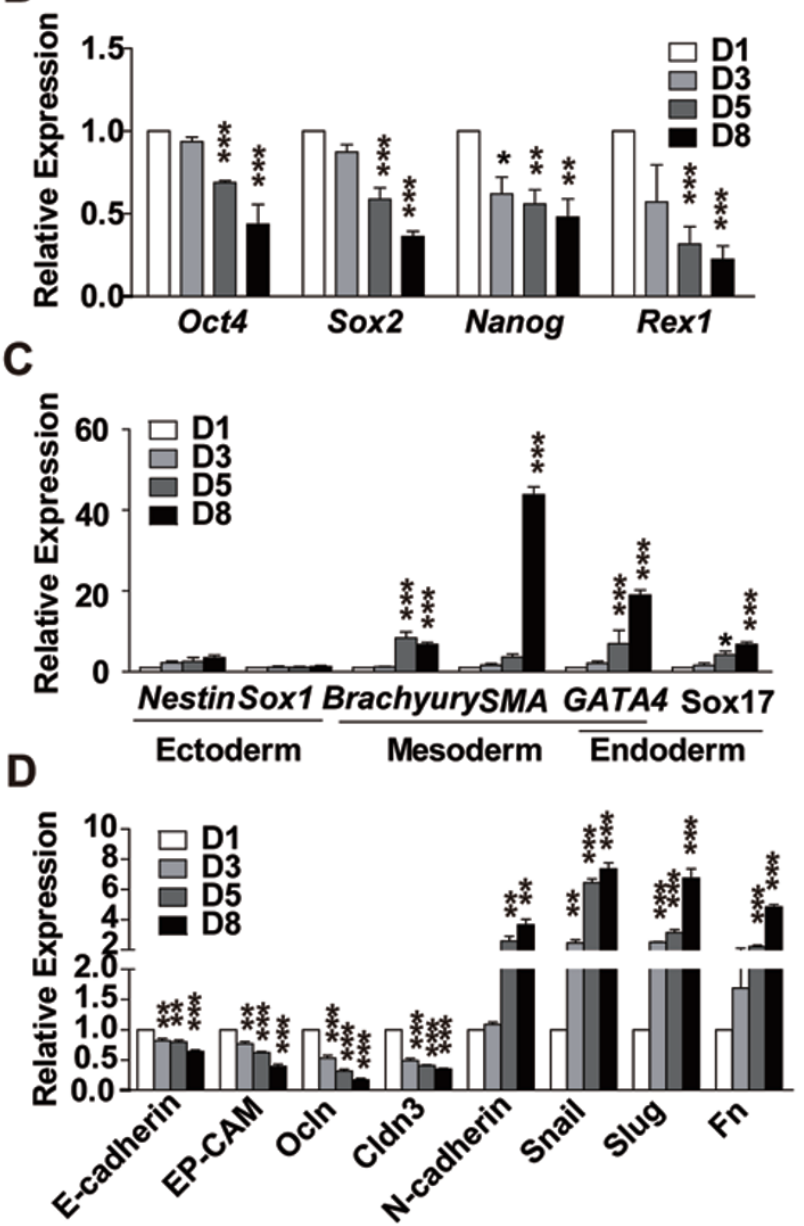

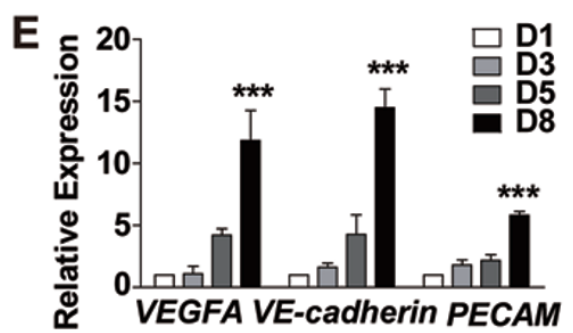

$\mathbf{F}$

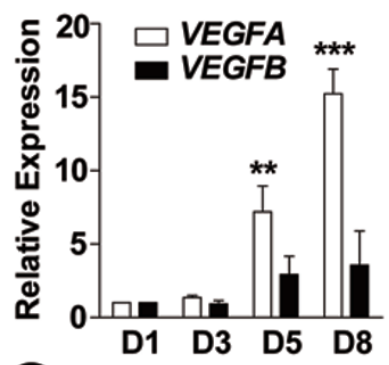

G

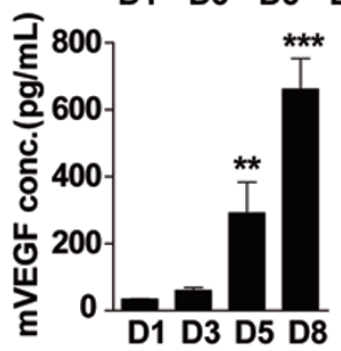

H

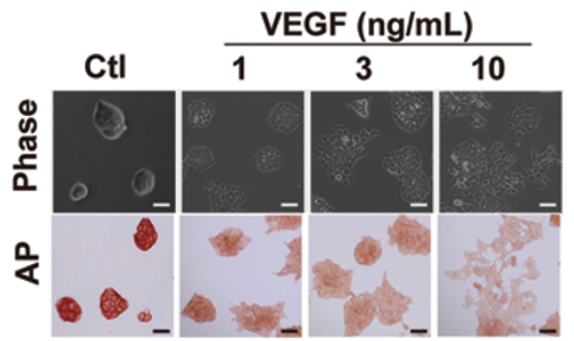

Figure $1 \mathrm{mESCs}$ differentiate toward meso-endoderm lineage spontaneously during long-term culture. (A) Morphology of E14 cells cultured in mES medium containing $1000 \mathrm{U} / \mathrm{ml}$ LIF without passage for 1-8 days. Medium was changed every day. Scale bar, $50 \mu \mathrm{m}$. (B-F) qRT-PCR analysis of pluripotency genes (B), lineage-specific genes (mesoderm: Brachyury and SMA, endoderm: GATA4, Sox17, ectoderm: Nestin and Sox1) (C), genes involved in EMT (D), vascular marker genes (E) and VEGF genes (F) in mESCs described in $\mathbf{A}$ on days 1, 3, 5 and 8. (G) ELISA analysis of VEGF secretion from mESCs described in A. (H) VEGF-induced morphology and alkaline phosphatase (AP) change in E14 cells. Scale bar, $50 \mu \mathrm{m}$. E14 cells were cultured in mES medium containing $1000 \mathrm{U} / \mathrm{ml}$ LIF for $48 \mathrm{~h}$, then vehicle or $1,3,10 \mathrm{ng} / \mathrm{ml} \mathrm{mVEGF}{ }_{164}$ was added into the medium for another $48 \mathrm{~h}$ prior to analysis. Data are mean $\pm \operatorname{SEM}(n=3) .{ }^{*} P<0.05,{ }^{* \star} P<0.01,{ }^{* \star *} P<0.001$ vs day 1 . Independent experiments were repeated at least three times. 
with shRNA in E14 cells significantly blocked the differentiation induced by LIF withdrawal (Supplementary information, Figure S2B-S2E), suggesting that PDGFB might induce differentiation in a paracrine fashion, while VEGFA in an autocrine way. Taken together, these data suggest that during long-term culture, mESCs secrete growth factors such as VEGFA, which promote cells to differentiate toward meso-endoderm lineages.

Blocking VEGF receptors with sunitinib promotes $m E S C$ self-renewal in the absence of LIF and de novo derivation of pluripotent ESCs

VEGF might promote spontaneous differentiation of mESCs in an autocrine way, thus we investigated whether blocking VEGF signaling with small-molecule inhibitors would help to maintain the self-renewal of mESCs. In mES media deprived of LIF, E14 cells showed spontaneous differentiation on day 3 (Figure 2A). $2 \mathrm{i}$ (PD0325901 and CHIR99021) effectively maintained the mESC morphology up to day 5 without passage, while sunitinib, a multi-targeted RTK inhibitor mainly inhibiting VEGF and PDGF receptors, showed a similar effect in maintaining the self-renewal of mESCs (Figure 2A). Dose-response study indicated that $1 \mu \mathrm{M}$ sunitinib was best in maintaining the undifferentiated state of mESCs in LIF-free condition (Supplementary information, Figure S3A). In the presence of LIF, both sunitinib and $2 \mathrm{i}$ were able to maintain the mESC morphology up to day 8 without passage (Supplementary information, Figure $\mathrm{S} 3 \mathrm{~B})$. In the absence of LIF, mESCs could be maintained for 3-4 passages, even though the alkaline phosphatase (AP) staining results revealed the loss of pluripotency at passage 1 (Figure 2B). Both sunitinib and $2 \mathrm{i}$ helped to maintain the mESC morphology and positive AP staining beyond passage 10 (Figure 2B). Immunocytochemistry data showed that mESCs maintained in sunitinib ${ }^{+}$LIF $^{-}$ medium expressed high levels of pluripotency markers, including Oct4, Nanog and SSEA1, in passages up to 10, while cells in the sunitinib ${ }^{-}$LIF $^{-}$medium completely lost the expression of these genes at passage 4 (Supplementary information, Figure S4A). Quantitative PCR results revealed that both sunitinib and $2 \mathrm{i}$ almost completely reversed the upregulation of lineage-specific genes, including SMA, Desmin, Nestin and VE-cadherin, and the loss of pluripotency genes, such as Oct4, Sox2, Nanog and Rex1, caused by the removal of LIF (Supplementary information, Figure S4B and S4C). And LIF deprivation-induced changes in the EMT-related genes, including E-cadherin, EP-CAM, Ocln, Cldn3, N-cadherin, Snail, Slug and $F n$, were also blocked by both sunitinib and $2 \mathrm{i}$ (Supplementary information, Figure S4D). Sunitinib $(1 \mu \mathrm{M})$ was also very effective in maintaining the self-renewal of iPSCs generated from OG2 mice carrying a transgenic GFP gene driven by Oct4 promoter in the absence of LIF (Supplementary information, Figure $\mathrm{S} 3 \mathrm{C})$.

To confirm the intact pluripotency, E14 cells cultured in medium containing sunitinib, $2 \mathrm{i}$ or LIF (passage 11) were induced to form embryoid body (EB) after removal of sunitinib, $2 \mathrm{i}$ or LIF. No difference was observed in EB formation between cells previously maintained in different media. qRT-PCR analysis revealed the upregulation of lineage-specific genes and downregulation of pluripotent genes in EBs formed by E14 cells previously maintained in all three media (Supplementary information, Figure S5A). For immunofluorescent staining, EBs were reseeded into gelatin-coated plates and cells spontaneously differentiating into three germ layers could be identified (Supplementary information, Figure $\mathrm{S} 5 \mathrm{~B})$. Stimulation by $\mathrm{mVEGF}_{164}(50 \mathrm{ng} / \mathrm{ml})$ promoted EB differentiation toward vascular cells with increased expression of vascular lineage markers, including SMA, PECAM and VE-cadherin (Supplementary information, Figure S5C and S5D). For in vivo differentiation, mESCs from passage 11 were injected into the thigh of SCID mice. Teratoma formation was observed after 3-4 weeks. $H \& E$ staining revealed that these tumors contain tissues derived from all three germ layers, including gastrointestinal-like epidermis structure (ectoderm), cartilage-like structure (mesoderm) and neural tube-like structure (endoderm; Figure 2C and Supplementary information, Table S1). Sunitinib seems to exert its anti-differentiation effect by blocking VEGF-induced ERK and GSK3 $\beta$ activation (Supplementary information, Figure S6A) rather than by direct activation of the Stat3 pathway like LIF (Supplementary information, Figure S6B).

Since the E14 cells were derived from 129/Ola mice with cream coat color, it would be difficult to assess the chimerism between E14 cells and ICR mice (with white coat color). Therefore, we generated mESCs from the blastocysts of C57BL/6 mice, which have black coat color, and cultured them in mES medium containing sunitinib $(1 \mu \mathrm{M})$ and $2000 \mathrm{U} / \mathrm{ml} \mathrm{LIF.} \mathrm{mESCs} \mathrm{were} \mathrm{later}$ expanded in mES medium containing only sunitinib (1 $\mu \mathrm{M})$ (Figure 2D). Cells from passages 6 and 11 were injected into the blastocysts of ICR mice, and chimeras were generated successfully (Figure 2E). Taken together, these data indicate that blocking VEGF signaling by sunitinib help to maintain the pluripotency of mESCs, and the effect is comparable to $2 \mathrm{i}$.

Knocking down VEGFR promotes long-term self-renewal of $m E S C$ s in the absence of LIF

Although mainly targeting VEGFRs and PDGFRs, 
A

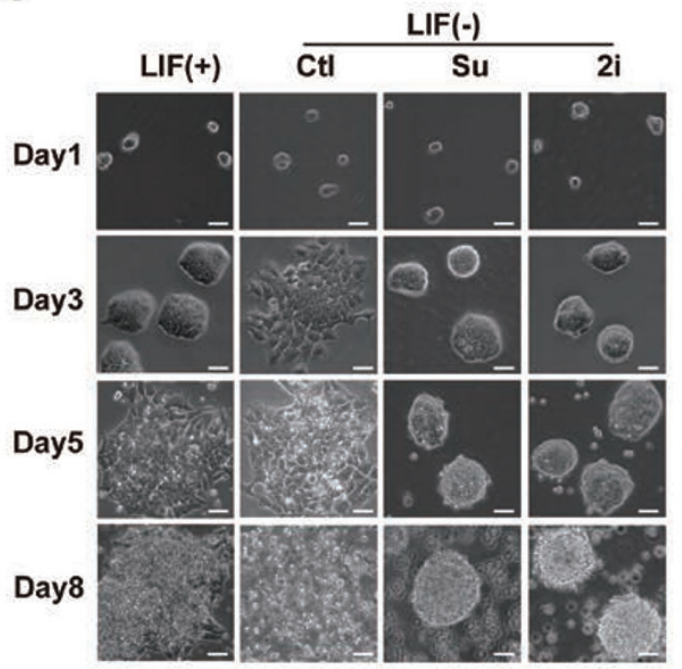

B

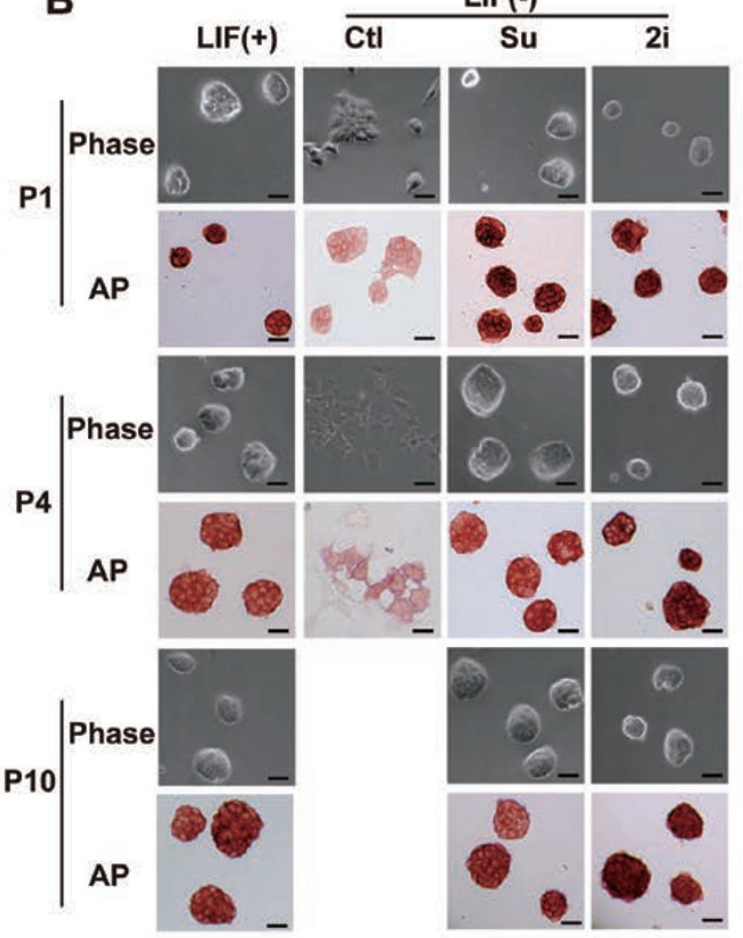

C

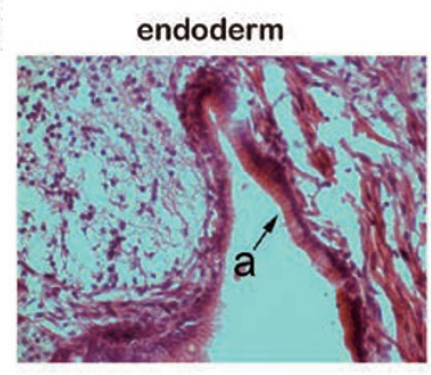

D

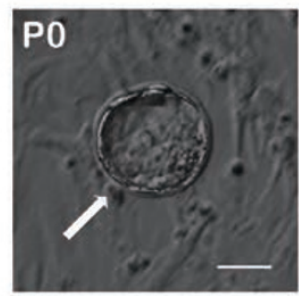

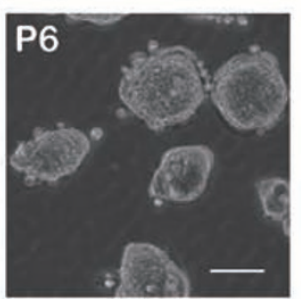

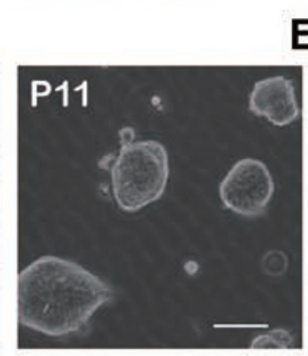

mesoderm

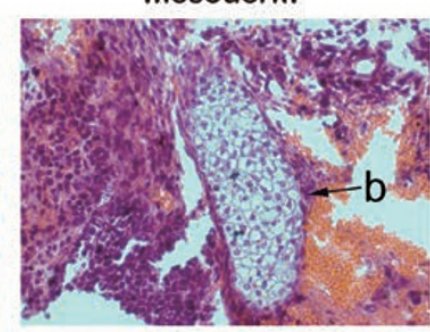

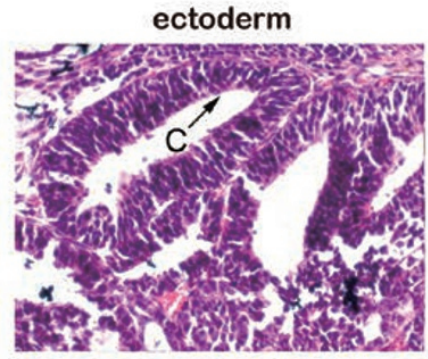

E

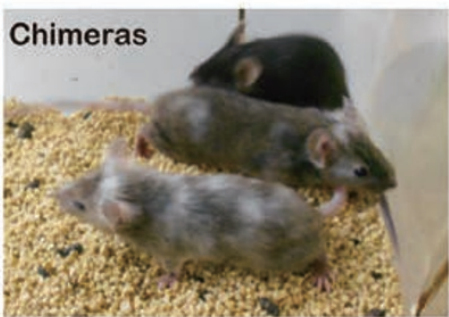

Figure 2 Sunitinib maintains the self-renewal and pluripotency of mESCs. (A) Morphology of E14 cells cultured in mES media containing $1000 \mathrm{U} / \mathrm{ml} \mathrm{LIF,} \mathrm{or} \mathrm{in} \mathrm{mES} \mathrm{media} \mathrm{without} \mathrm{LIF} \mathrm{but} \mathrm{containing} \mathrm{2i} \mathrm{(3 \mu M} \mathrm{PD0325901} \mathrm{and} 3 \mu \mathrm{M}$ CHIR99021) or sunitinib (Su, $1 \mu \mathrm{M}$ ), for 1-8 days. Media were changed every day. Scale bar, $50 \mu \mathrm{m}$. (B) Morphology and alkaline phosphatase (AP) staining of E14 cells at passages 1, 4 and 10 maintained in mES media containing $1000 \mathrm{U} / \mathrm{ml} \mathrm{LIF}$, or in mES media without LIF but containing 2i (3 $\mu$ M PD0325901 and $3 \mu \mathrm{M}$ CHIR99021) or sunitinib $(1 \mu \mathrm{M})$. Scale bar, $50 \mu \mathrm{m}$. (C) H\&E staining of teratomas generated with E14 cells maintained with sunitinib (passage 11). Typical structures of the three embryonic germ layers are shown as follows: a, gastrointestinal-like epithelium (endoderm); b, cartilage-like structure (mesoderm); c, neural tube-like structure (ectoderm). Scale bars, $20 \mu \mathrm{m}$. (D) Blastocyst outgrowth (P0, the arrow) on feeder cells and mES medium containing sunitinib and $2000 \mathrm{U} / \mathrm{ml}$ LIF. mESCs were subsequently maintained in mES media containing sunitinib for multiple passages. Scale bar, $20 \mu \mathrm{m}$. (E) Chimeric mice generated with mESCs derived and maintained with sunitinib (passage 6). 
sunitinib also blocks other RTKs including KIT, FLT3, and RET [19]. To avoid the possible off-target effect of sunitinib, we designed shRNAs specifically targeting $V E G F R 1$ and $V E G F R 2$, two main receptors convoying VEGFA signaling [20]. Both receptors were expressed in mESCs and the effectiveness of shRNAs was confirmed with quantitative RT-PCR and western blot analysis (Figure $3 \mathrm{~A}$ and $3 \mathrm{~B}$ ). When LIF was deprived for 2 days, E14 cells expressing scrambled shRNA almost completely lost the compact colonial morphology, while knocking down either VEGFR1 or 2 helped maintain the mESClike morphology (Figure 3C). The reduced expression of
A

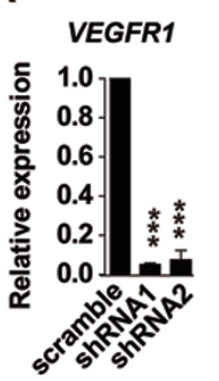

B

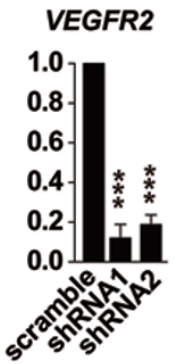

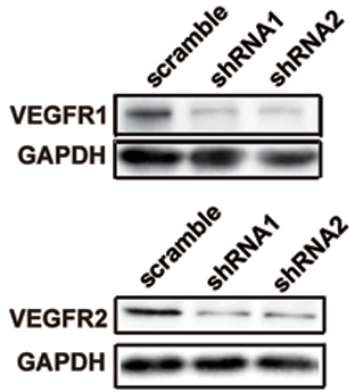

C

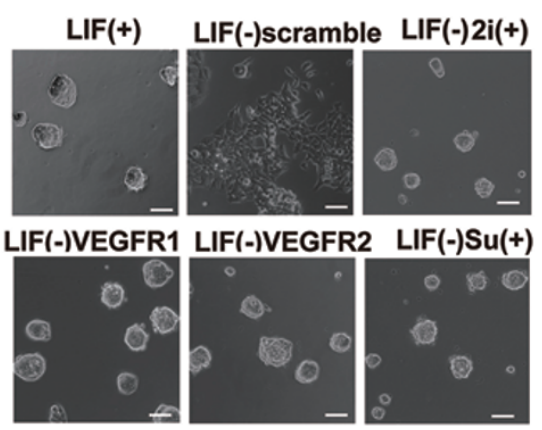

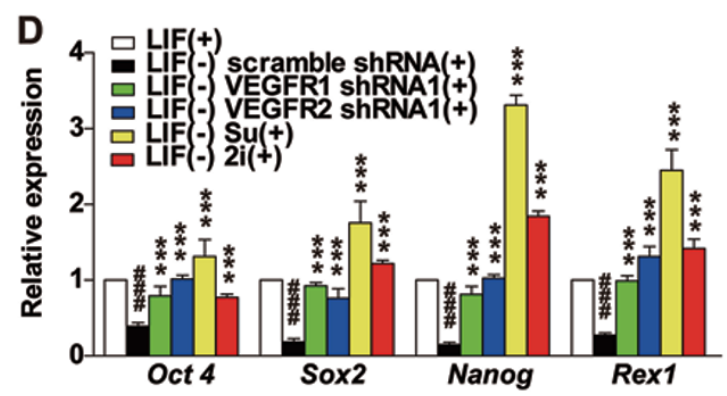

$\mathbf{F}$

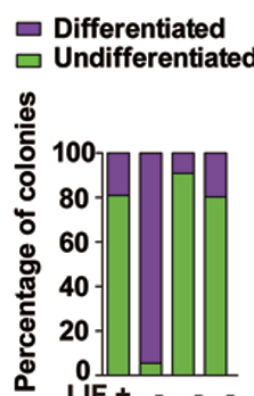

scramble shRNA : + -

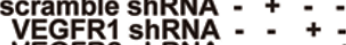

VEGFR1 shRNA - - + +
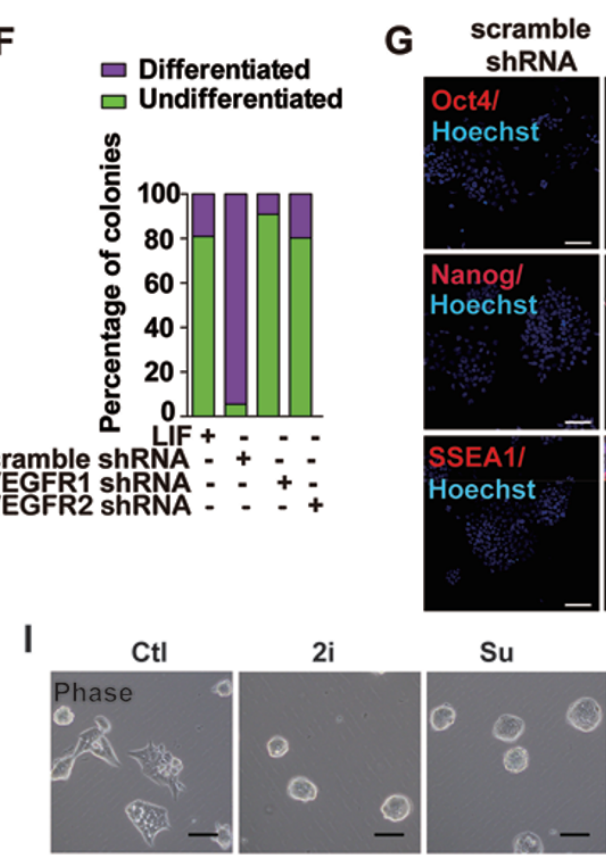

ĀP
$2 i$

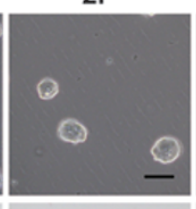

Su

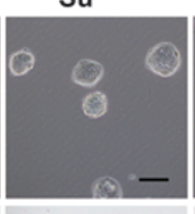

E

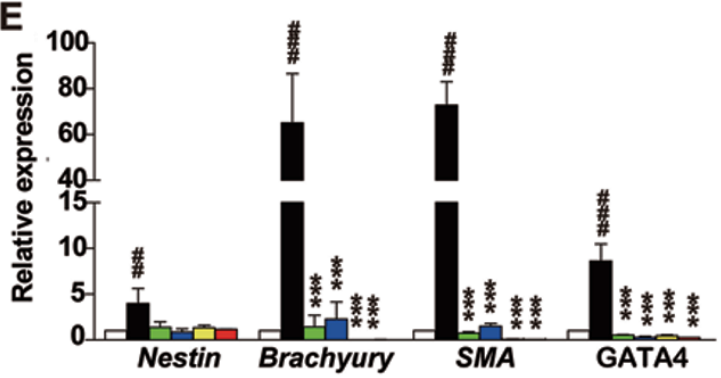

VEGFR
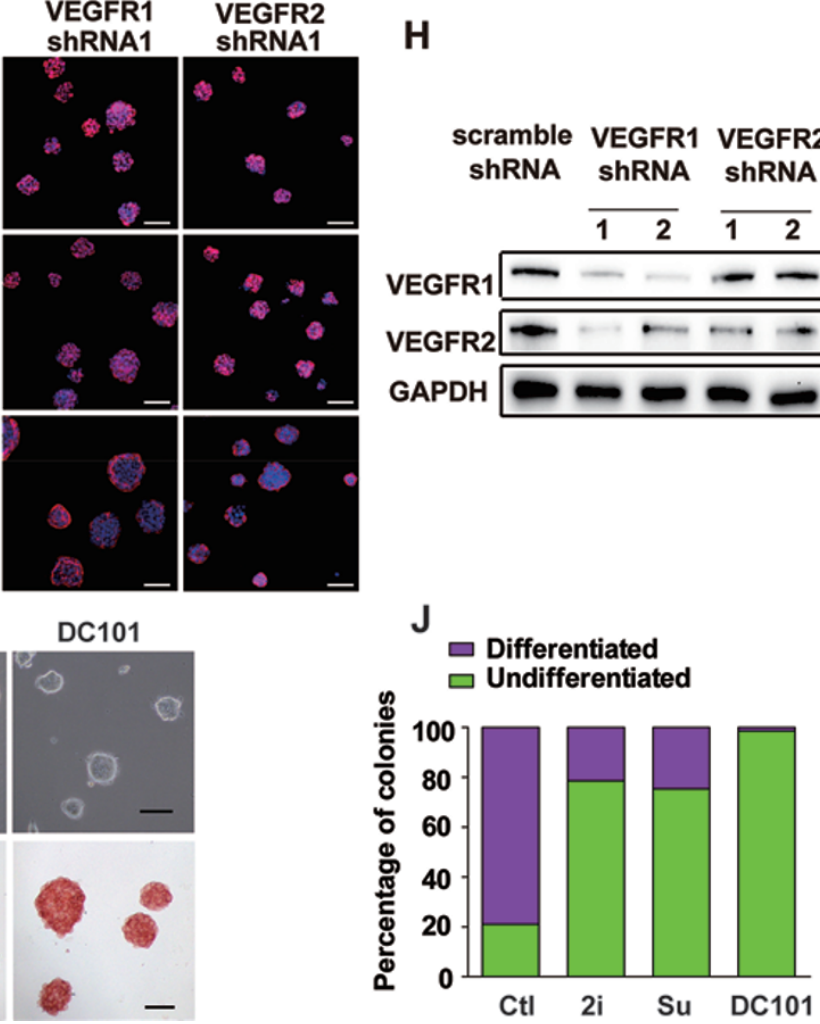
pluripotency genes and the elevated expression of lineage-specific genes caused by LIF removal could also be reversed by knockdown of either VEGFR1 or 2 (Figure $3 \mathrm{D}$ and $3 \mathrm{E}$ ). About $80 \%$ of E14 colonies maintained in LIF-containing medium were AP positive, while $<10 \%$ of E14 cells expressing scramble shRNA remained to be AP positive after LIF removal (Figure 3F). Knocking down either VEGFR 1 or 2 restored the percentage of AP-positive colonies to more than $80 \%$ (Figure $3 \mathrm{~F}$ ). Immunocytochemistry data also showed that VEGFR-knockdown E14 cells (passage 4) maintain high levels of pluripotency markers, including Oct4, Nanog and SSEA1, in LIF-free mES medium, while the scramble shRNA did not prevent the loss of all these markers (Figure 3G). It was intriguing to find that knocking down either VEGFR1 or 2 almost completely resembled the effect of sunitinib and blocked the spontaneous differentiation induced by LIF removal. It has been reported that VEGFR1 is a critical determinant of VEGFR2 abundance, while VEGFR2 is the key receptor directly responsible for endothelial cell signaling stimulated by VEGF [21]. Therefore, we examined whether knockdown of one VEGFR affect the level of the other VEGFR. In agreement with the previous report, knocking down VEGFR1 also reduced the expression of VEGFR2, but knocking down $V E G F R 2$ did not affect the expression of VEGFR1 (Figure 3H).

To further confirm the role of VEGFR2 in mESC differentiation, DC101, a neutralizing antibody of VEGFR2 [22], was tested along with $2 \mathrm{i}$ and sunitinib in mESCs cultured without LIF. As demonstrated in Figure 3I and 3J, DC101 blocked mESC differentiation induced by LIF withdrawal as effectively as sunitinib and $2 \mathrm{i}$. Taken together, these data indicate that autocrine activation of VEGFRs, especially VEGFR2, in mESCs is one of the major forces that promote the exit from self-renewal and entry into lineage commitment.
Hypoxia-inducible factor alpha and endoplasmic reticulum stress are involved in the VEGF production in mESCs during long-term culture

Hypoxia-inducible factor alpha (HIF $1 \alpha)$ is one of the major factors that regulate VEGF expression and angiogenesis in cancers [23]. There is still controversy in the roles of hypoxia in maintaining the self-renewal of ESCs. Some have reported that hypoxia promotes ESC propagation [24] and facilitates the generation of iPSCs [25], while others found that hypoxic priming of mESCs accelerates vascular-lineage differentiation through HIF1-dependent mechanisms [26, 27]. Nevertheless, we found that both the mRNA and protein level of HIF $1 \alpha$ increased significantly on day 3 during the long-term culture without passage but with daily medium change (Figure 4A and 4B). In LIF-free mES media, HIF $1 \alpha$ inducers $\mathrm{CoCl}_{2}$ [28] and ML228 [29] led to elevated expression of VEGFA and promoted cell differentiation further (Figure 4E-4G). In contrast, KC7F2 [30], a selective HIF1 $\alpha$ inhibitor, reduced the expression of VEGFA (Figure 4G) and blocked the spontaneous differentiation caused by the removal of LIF for 2 days (Figure 4E and 4F). Sunitinib and $2 \mathrm{i}$ also reduced the expression of VEGFA (Figure $4 \mathrm{G}$ ) and differentiation induced by LIF deprivation (Figure $4 \mathrm{E}$ and $4 \mathrm{~F}$ ).

Endoplasmic reticulum (ER) stress is another major pathway that regulates the expression of VEGF under adverse environmental conditions [31]. It has been reported that hESCs must resolve ER stress related to culture conditions to survive and maintain pluripotency [32]. ER stress has also been reported to participate in ESC differentiation induced by retinoic acid [33]. Therefore, we further investigated the expression of genes related to ER stress during long-term culture of mESCs. Quantitative PCR indicated that the expression of several genes involved in ER stress, including $X B P 1$ and splXBP1 (an alternative splicing form of XBP1 in re-

Figure 3 VEGFR knockdown recapitulates the effect of sunitinib. (A, B) Validation of the shRNAs targeting VEGFRs by qRTPCR and western blot. (C) Morphology of E14 cells expressing the indicated shRNA cultured in mES media without LIF. Cells treated with sunitinib or $2 \mathrm{i}$ were used as positive control. Scale bar, $50 \mu \mathrm{m}$. (D, E) qRT-PCR analysis of pluripotency genes (Oct4, Sox2, Nanog, Rex1) (D) and lineage-specific genes (mesoderm: Brachyury and SMA, endoderm: GATA4, ectoderm: Nestin) (E) in cells expressing the indicated shRNA cultured in mES media without LIF (passage 4). Cells treated with sunitinib or LIF were used as positive control. Data are mean SEM $(n=3) .{ }^{\# \#} P<0.01,{ }^{\# \#} P<0.001$ vs LIF $(+)$ condition; ${ }^{*} P<0.05$, ${ }^{* *} P<0.01,{ }^{* * *} P<0.001$ vs scramble shRNA. (F) E14 cells expressing the indicated shRNA were cultured in LIF-free mES media for 3 days at an initial density of 10000 cells/well in 24-well plate. The percent of undifferentiated and differentiated colonies were calculated according to AP staining and morphology. Independent experiments were repeated at least three times. (G) Immunofluorescence staining of pluripotency markers (Oct4, Nanog, SSEA1) in cells described in E (passage 4). Cell nuclei were stained with Hoechst. Scale bar, $80 \mu \mathrm{m}$. (H) Western blot analysis of VEGFR1 and 2 in cells transfected with scramble shRNA, or shRNAs targeting VEGFR1 or 2 for $48 \mathrm{~h}$. (I) Morphology and AP staining of E14 cells cultured in mES medium without LIF but containing $2 \mathrm{i}$ ( $3 \mu \mathrm{M}$ PD0325901 and $3 \mu \mathrm{M}$ CHIR99021), sunitinib $(1 \mu \mathrm{M})$ or DC101 (20 $\mu \mathrm{g} / \mathrm{ml})$ for 3 days. (J) The percent of undifferentiated and differentiated colonies corresponding to $\mathbf{I}$. 
sponse to ER stress), Ire 1 $\alpha$, Grp 78 and $C H O P$, increased with varying degrees from day 1 to day 8 (Figure 4C). Among them, splXBP 1 and $C H O P$ increased dramatically on day 5 and the protein levels of XBP1 and CHOP increased in a time-dependent manner and peaked on day 6 (Figure 4D). In the absence of LIF, ER stress inducers thapsigargin, tunicamycin and ionomycin [34] lead to more severe differentiation and higher expression of ER
A

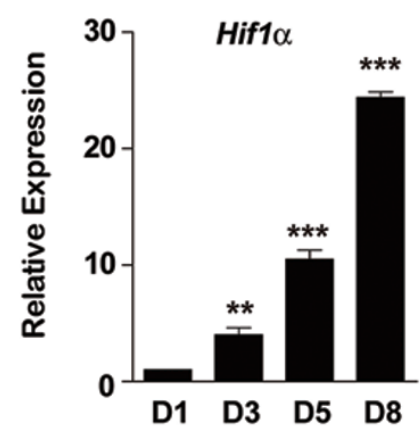

B

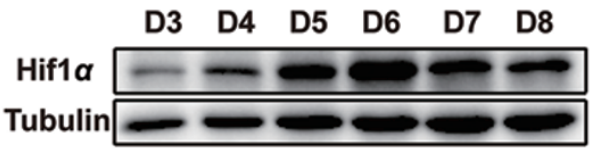

C

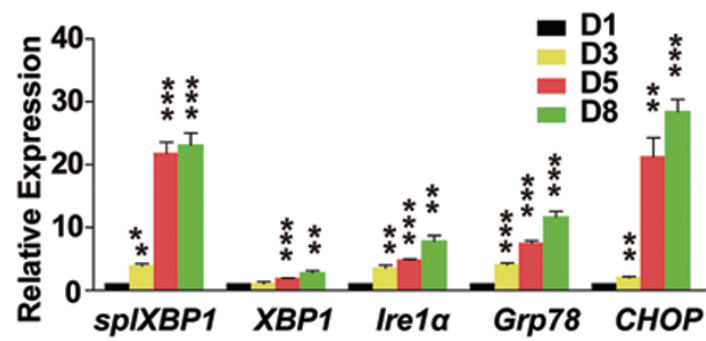

D

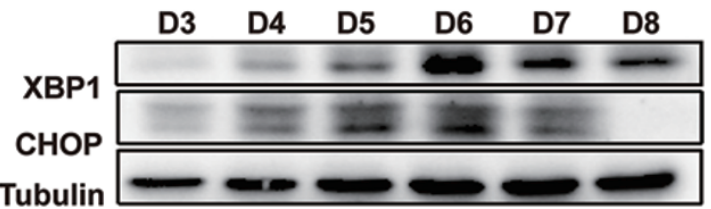

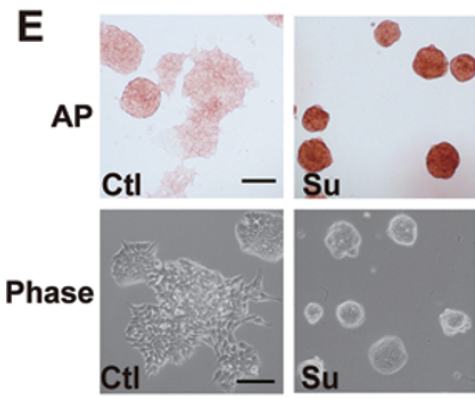

$\mathbf{F}$

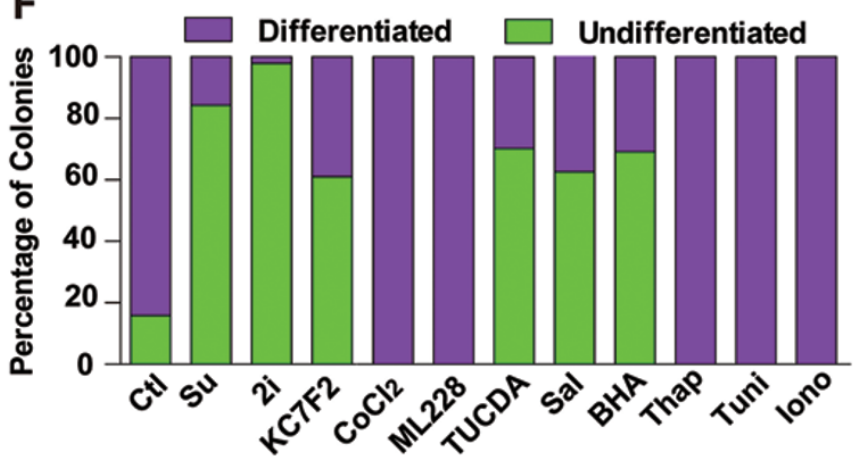

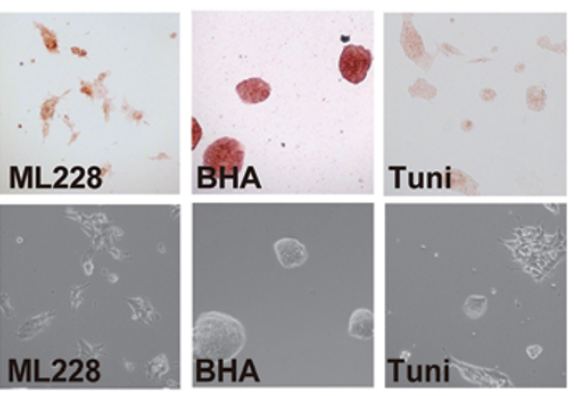

G

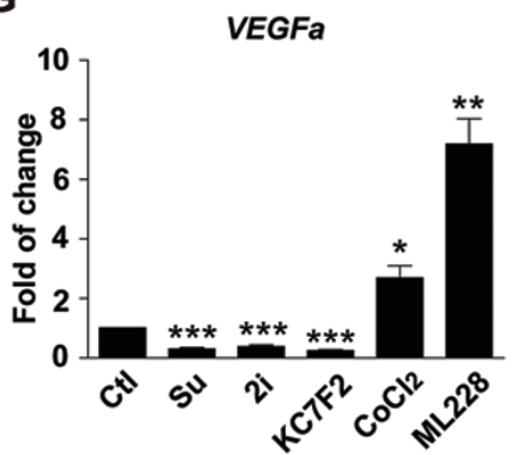

H

$\square$ Ctl $\square$ TUCDA $\square$ Thap

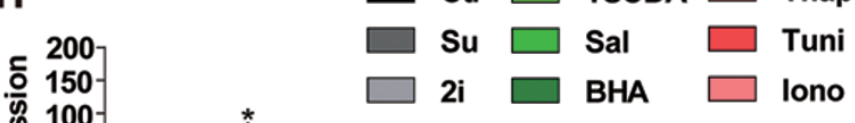

8

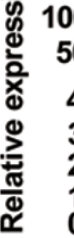

BHA

Iono

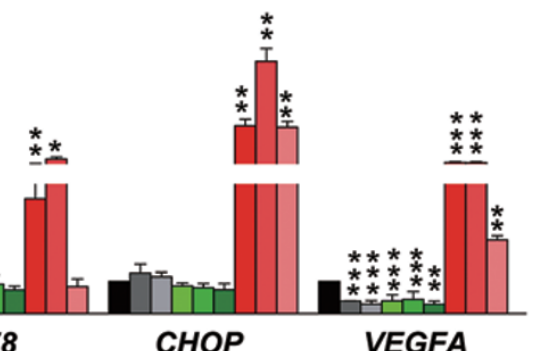


stress-related genes and VEGFA (Figure 4E, $4 \mathrm{~F}$ and $4 \mathrm{H}$ ), while tauroursodeoxycholic acid, salubrinal and BHA [34], the ER stress inhibitors, blocked the expression of ER stress-related genes and VEGFA caused by the removal of LIF (Figure 4H), and thus prevented cell differentiation (Figure 4E and 4F).

It was reported that the retina of LIF-knockout mice displayed increased microvessel density due to increased VEGF expression [35]. We also found that LIF suppressed the expression of VEGFs in E14 cells during long-term culture (Supplementary information, Figure S7A). And such effect was likely due to the suppressed expression of HIF $1 \alpha$ and ER stress-related genes by LIF (Supplementary information, Figure S7B).

Taken together, these data suggest that both HIF $1 \alpha$ and ER stress are involved in the expression of VEGFA in $\mathrm{mESCs}$, blocking these pathways is beneficial for the maintenance of pluripotency and self-renewal of mESCs. Interestingly, blocking the VEGF pathways with sunitinib decreased the expression of ER stress-related genes and VEGFA (Figure $4 \mathrm{G}$ and $4 \mathrm{H}$ ), indicating the possible existence of a feed-forward mechanism between VEGF and HIF $1 \alpha /$ ER stress pathways. LIF may also promote self-renewal of mESCs by reducing $V E G F$ expression via downregulation of HIF $1 \alpha$ and ER stress pathways.

\section{Sunitinib promotes the generation of mouse iPSCs with} four or reduced numbers of transcription factors

A number of small molecules, including 2i, pluripotin, $\mathrm{BIO}$ and PKC inhibitors, which support ESC self-renewal by inhibiting signal pathways involved in differentiation, have been reported to enhance the generation of iPSCs. Therefore, we investigated whether sunitinib can also promote reprogramming. Using a 96-well-platebased screening system with OG2 MEFs carrying a transgenic Oct4 promoter-driven GFP reporter [36], we found in 4 factor (4F: Oct4, Sox2, Klf4 and c-Myc)-mediated reprogramming, $1 \mu \mathrm{M}$ sunitinib (added from day
3 to 14 ) was able to induce significantly more $\mathrm{GFP}^{+}$colonies ( $\sim 8$-fold increase) than vehicle control (Figure $5 \mathrm{~A}$ ). We also treated the 4F-transduced MEF cells with $1 \mu \mathrm{M}$ sunitinib for various durations starting from day 3. Sunitinib started to show beneficial effect after 5-day treatment (day 3-8). In fact, the longer the treatment duration, the better the results (Figure 5B), supporting the notion that sunitinib can promote the self-renewal of pluripotent stem cells. Indeed, sunitinib also supported the propagation of iPSCs in the absence of LIF (Supplementary information, Figure S3C).

Combinations of small molecules have been reported to facilitate the generation of iPSCs with reduced numbers of transcription factors. In $2 \mathrm{~F}$ (Oct4 and Klf4)-transduced MEFs, sunitinib $(1 \mu \mathrm{M})$ facilitated iPSC generation alone or in combination with RepSox $(1 \mu \mathrm{M})$, VPA (0.5 mM), CHIR99021 (3 M) and parnate $(2 \mu \mathrm{M}$; Figure 5C). More remarkably, sunitinib $(1 \mu \mathrm{M})$ alone was able to enhance the efficiency of $1 \mathrm{~F}$ (Oct4)-mediated reprogramming (Figure 5D). Sunitinib also doubled the number of $\mathrm{GFP}^{+}$colonies induced by Oct 4 in combination with RepSox $(1 \mu \mathrm{M})$, VPA $(0.5 \mathrm{mM})$, CHIR99021 $(3 \mu \mathrm{M})$ and parnate $(2 \mu \mathrm{M}$; Figure $5 \mathrm{D})$. Knockdown of VEGFR 1 or VEGFR2 also facilitated 4F-mediated reprogramming (Supplementary information, Figure S8G).

A series of iPSC lines were established by using 4F, $3 \mathrm{~F}, 2 \mathrm{~F}$ or $1 \mathrm{~F}$ plus sunitinib. PCR analysis of genomic DNA confirmed the integration of correct viral factors in these iPSC lines (Supplementary information, Figure S8A). Quantitative RT-PCR revealed the reactivation and expression of the endogenous Oct4, Sox2, Nanog and Rexl (Supplementary information, Figure S8B) and the silencing of viral genes (Supplementary information, Figure S8C). 1F- and 2F-iPSCs were further characterized. These iPSCs maintain $\mathrm{GFP}^{+}$- and ES-like morphology, and express typical pluripotency markers, such as AP, SSEA1 and Nanog (Figure 5E and Supplementary information, Figure S8D). Bisulfite genomic sequenc-

Figure 4 Role of HIF1 $\alpha$ and ER stress in VEGF expression and pluripotency of mESCs. (A, B) qRT-PCR (A) and western blot analysis (B) of HIF1 $\alpha$ in E14 cells cultured in mES media containing $1000 \mathrm{U} / \mathrm{ml}$ LIF for 1-8 days. Tubulin served as a loading control for western blot. (C, D) qRT-PCR (C) and western blot analysis (D) of genes involved in ER stress in E14 cells cultured in mES medium containing $1000 \mathrm{U} / \mathrm{ml}$ LIF for 1-8 days. (E) Morphology (bottom) and AP staining (top) of E14 cells cultured in mES medium without LIF but containing sunitinib $(1 \mu \mathrm{M}), 2 \mathrm{i}, \mathrm{ML} 228(1 \mu \mathrm{M}), \mathrm{KC} 7 \mathrm{~F} 2(1 \mu \mathrm{M}), \mathrm{BHA}(10 \mu \mathrm{M})$ or tunicamycin (Tuni, $2.5 \mu \mathrm{g} / \mathrm{ml}$ ) for $48 \mathrm{~h}$. Scale bar, $50 \mu \mathrm{m}$. (F) Statistical data of the percent of undifferentiated and differentiated colonies in E14 cells cultured in LIF-free mES medium containing the indicated compounds for 3 days, including sunitinib, 2i, KC7F2 $(1 \mu \mathrm{M}), \mathrm{CoCl}_{2}(30 \mu \mathrm{M}), \mathrm{ML} 228(1 \mu \mathrm{M})$, TUCDA $(0.1 \mathrm{mM})$, salubrinal (Sal, $\left.10 \mu \mathrm{M}\right), \mathrm{BHA}(10 \mu \mathrm{M})$, thapsigargin (Thap, $0.4 \mu \mathrm{M}$ ), tunicamycin (Tuni, $2.5 \mu \mathrm{g} / \mathrm{ml}$ ) and ionomycin (Iono, $3.6 \mu \mathrm{M}$ ). (G) qRT-PCR analysis of VEGFA in E14 cells cultured in LIF-free mES medium containing sunitinib, $2 \mathrm{i}$ or the indicated HIF1 $\alpha$ regulators (same concentrations as in F) for 3 days. Data are mean \pm SEM $(n=3) .{ }^{*} P<0.05,{ }^{* *} P<0.01,{ }^{* * *} P<0.001$ vs control. (H) qRT-PCR analysis of the expression of ER stress-related genes and VEGFA in E14 cells cultured in LIF-free mES medium containing sunitinib, $2 i$ or the indicated ER stress regulators (same concentrations as in $\mathbf{F}$ ) for 3 days. Data are mean $\pm \operatorname{SEM}(n=3),{ }^{*} P<0.05,{ }^{* *} P<0.01,{ }^{* * *} P<0.001$ vs control. Independent experiments were repeated at least three times. 
ing of the Oct4 and Nanog promoters revealed that both were demethylated in $1 \mathrm{~F}$-iPSCs as in mESCs, while in MEFs they were both hypermethylated (Supplementary information, Figure S8E). Intramuscular injection of the 1F-iPSCs into SCID mice led to teratoma formation in 3-4 weeks, containing tissues derived from all three germ layers, including epidermis structure (ectoderm), muscle and cartilage structure (mesoderm) and epithelium tube structure (endoderm; Figure 5F). The 1F- and 2F-iPSCs were also able to generate chimera mice when injected into the blastocysts of ICR mice (Figure 5G and Supplementary information, Figure S8F), indicating that the
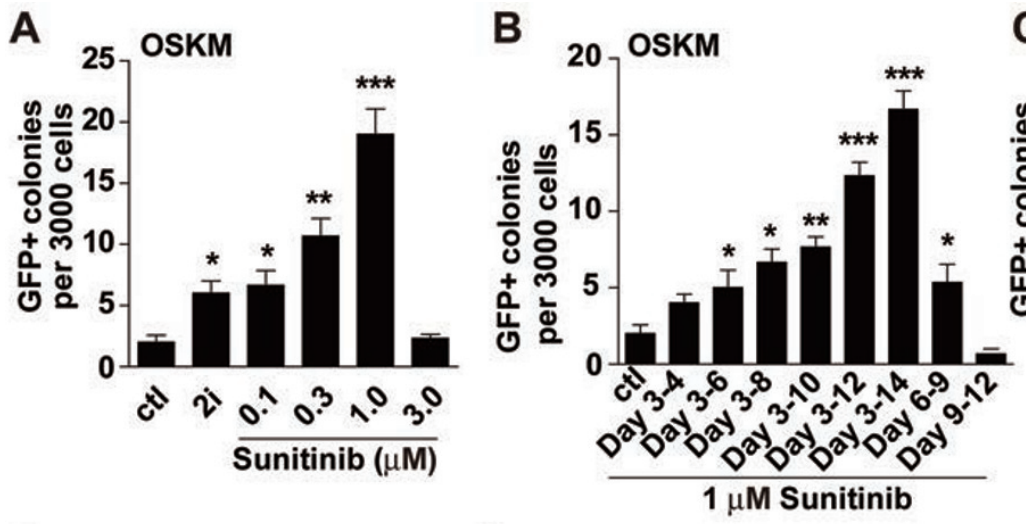

E

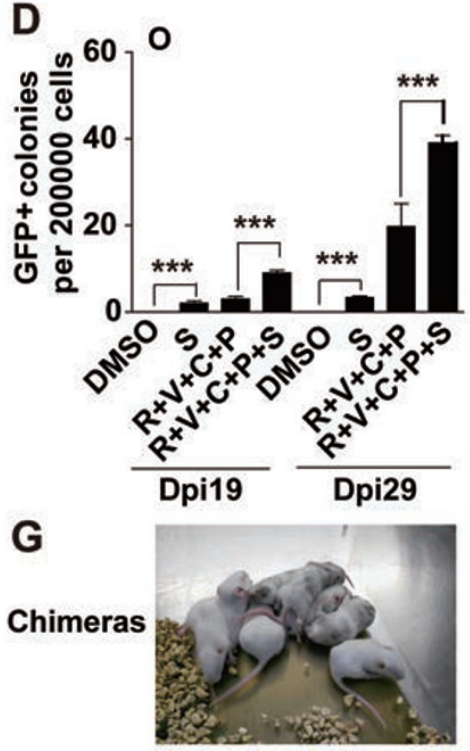

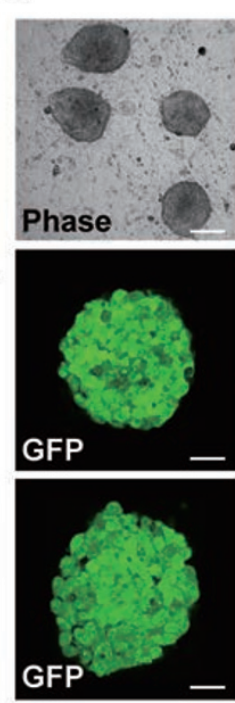

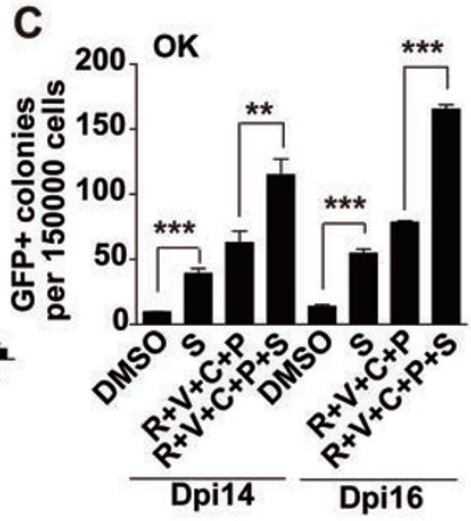

$\mathbf{F}$
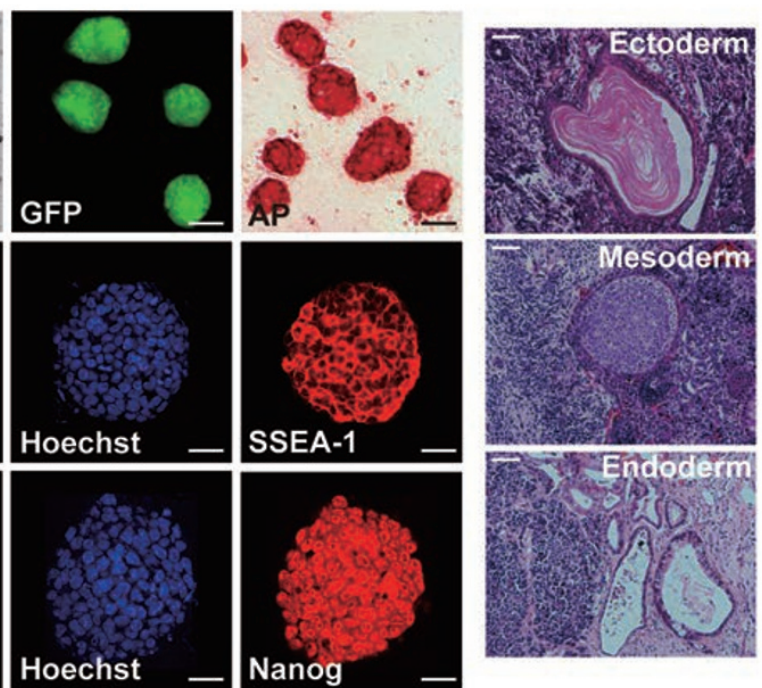

SSEA-1

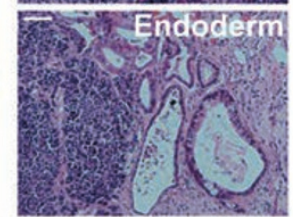

Figure 5 Sunitinib promotes reprogramming of somatic cells. (A) Dose response of sunitinib in 4F-mediated iPSC induction. Sunitinib at the indicated concentration was added into 3000 4F-transduced MEFs from day 3 to day 14. (B) Time response of sunitinib in iPSC induction. Sunitinib $(1 \mu \mathrm{M})$ was added at the indicated time periods, and GFP ${ }^{+}$colonies were counted on day 14. Data are mean $\operatorname{SEM}(n=3)$. ${ }^{*} P<0.05$, ${ }^{* *} P<0.01$, ${ }^{* *} P<0.001$ vs control. Independent experiments were repeated at least three times. (C) Sunitinib facilitated iPSC generation from $2 \mathrm{~F}(\mathrm{OK})$-transduced MEFs alone or in combination with several known chemicals. For OK-mediated reprogramming, 150000 OK-infected cells in 6-well plates were treated with 1 $\mu \mathrm{M}$ sunitinib (S) alone or various combinations of compounds (including $1 \mu \mathrm{M} \mathrm{R}$, RepSox; $0.5 \mathrm{mM} \mathrm{V,} \mathrm{VPA;} 3 \mu \mathrm{M} \mathrm{C}$, CHIR and $2 \mu \mathrm{M}$ P, Parnate) from day 3 to day 14. (D) Sunitinib facilitated iPSC generation from Oct4-transduced MEFs alone or in combination with several known chemicals. For Oct4-mediated reprogramming, 200000 Oct4-infected cells in 6-well plates were treated with $1 \mu \mathrm{M}$ sunitinib (S) alone or the indicated combinations of compounds from day 3 to day 14 . GFP ${ }^{+}$colonies were counted on day 19 and day 29. (E) Top: morphology, GFP expression and AP staining in iPSC clones induced with 1F (Oct4) plus sunitinib $(1 \mu \mathrm{M})$. Scale bar, $50 \mu \mathrm{m}$. Middle and bottom: immunofluorescent staining of SSEA-1 and Nanog in the same clone. Scale bar, $10 \mu \mathrm{m}$. (F) HE-stained sections of teratomas formed with iPSC clone described in E. Typical structures of the three embryonic germ layers are shown: epidermis (ectoderm), muscle and cartilage (mesoderm) and epithelium (endoderm). Scale bar, $20 \mu \mathrm{m}$. (G) chimeric mice produced with iPSC clone described in $\mathbf{E}$. 
iPSCs generated with sunitinib were pluripotent.

Other RTK inhibitors also facilitate the self-renewal of $m E S C$ and the generation of iPSCs

Apart from sunitinib, a number of RTK inhibitors whose targets include VEGFR and PDGFR have been developed and some have been used as anti-neoplastic drugs [19]. We tested whether these drugs or compounds could also be used to maintain the self-renewal of mESCs and facilitate factor-mediated reprogramming. E14 cells were seeded into 96-well plates at a density of 1000 cells per well in mES medium and allowed to grow with LIF for 1 day, and then media were changed into $\mathrm{mES}$ media without LIF but containing various compounds for 2 days. Then, cells were stained with AP staining kit. Cell colonies could be characterized into differentiated or undifferentiated categories according to the morphology and AP activity. All of the 10 RTK inhibitors, including axitinib, sorafenib, dovitinib, lenvatinib, motesanib, nintedanib, vandetanib, pazopanib, tivozanib and semaxinib, showed beneficial effect in maintaining the self-renewal of mESCs, with sunitinib and dovitinib being the most effective (Figure 6A). We also tested these compounds in our 96-well-plate-based iPSC induction systems with 4 factors, and all compounds significantly enhanced $4 \mathrm{~F}$-mediated reprogramming at their optimal concentrations, with sunitinib, dovitinib, axitinib and vandetanib showing the best results (Figure 6B).

\section{Discussion}

ESCs are isolated from the inner cell mass (ICM) of

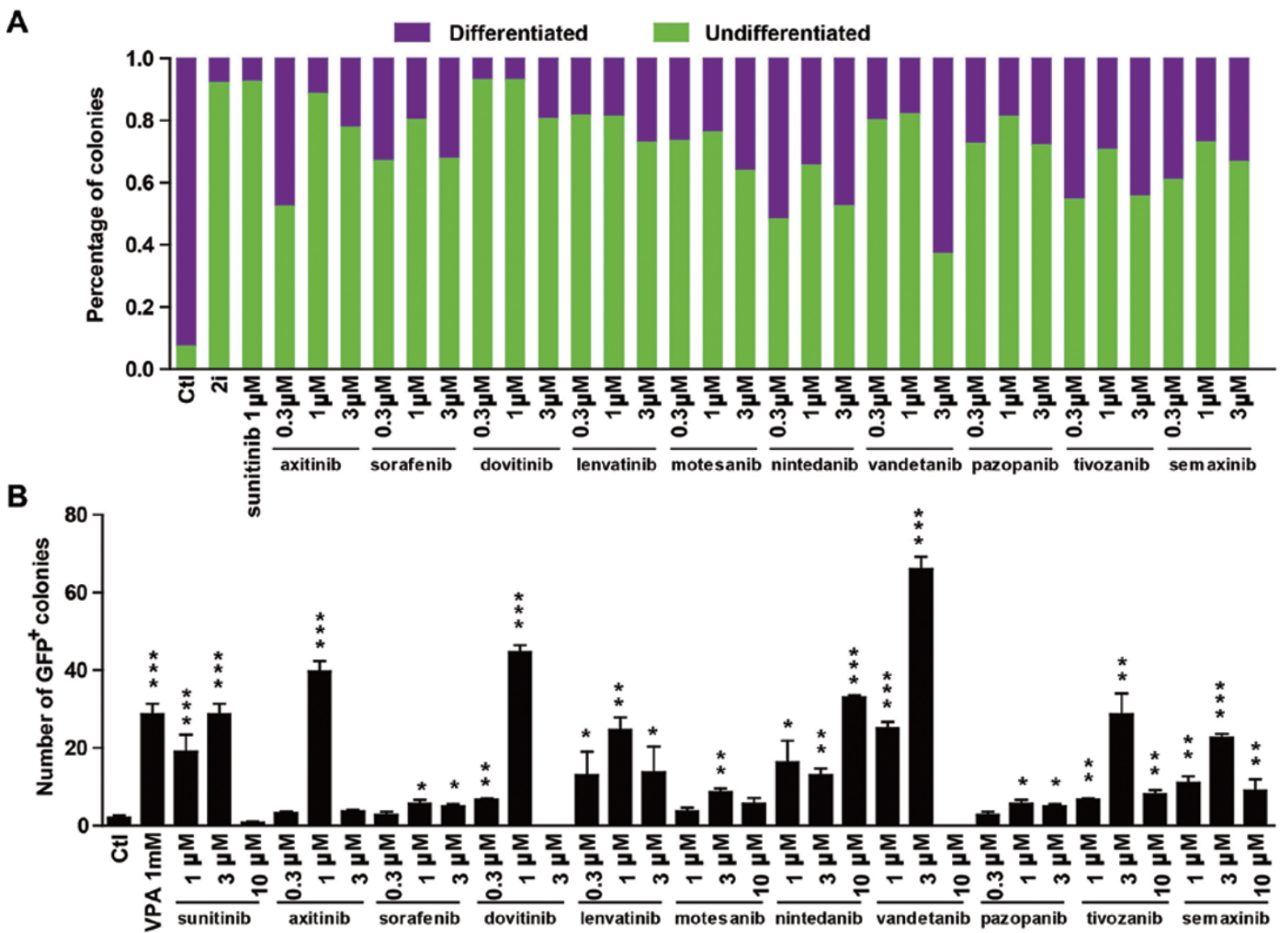

Figure 6 Other RTK inhibitors targeting VEGFRs facilitate the self-renewal of mESCs and the generation of iPSCs. (A) E14 cells were cultured in LIF-free mES media containing sunitinib or other inhibitors at the indicated concentrations for 3 days. The initial cell density was 1000 cells/well in 96-well plate. The percent of $\mathrm{AP}^{+}$(undifferentiated) and $\mathrm{AP}^{-}$(differentiated) colonies were calculated. 2i culture was used as a positive control. (B) MEFs transduced with Yamanaka 4 factors were treated with sunitinib or the indicated inhibitors (added from day 3 to day 14 ) and $\mathrm{GFP}^{+}$colonies were counted on day 14. VPA (1 $\mathrm{mM}$, added from day 3 to day 8 ) treatment was used as a positive control. Data are mean $\pm \operatorname{SEM}(n=3)$. ${ }^{*} P<0.05,{ }^{*} P<0.01$, ${ }^{* * *} P<0.001$ vs vehicle control. 


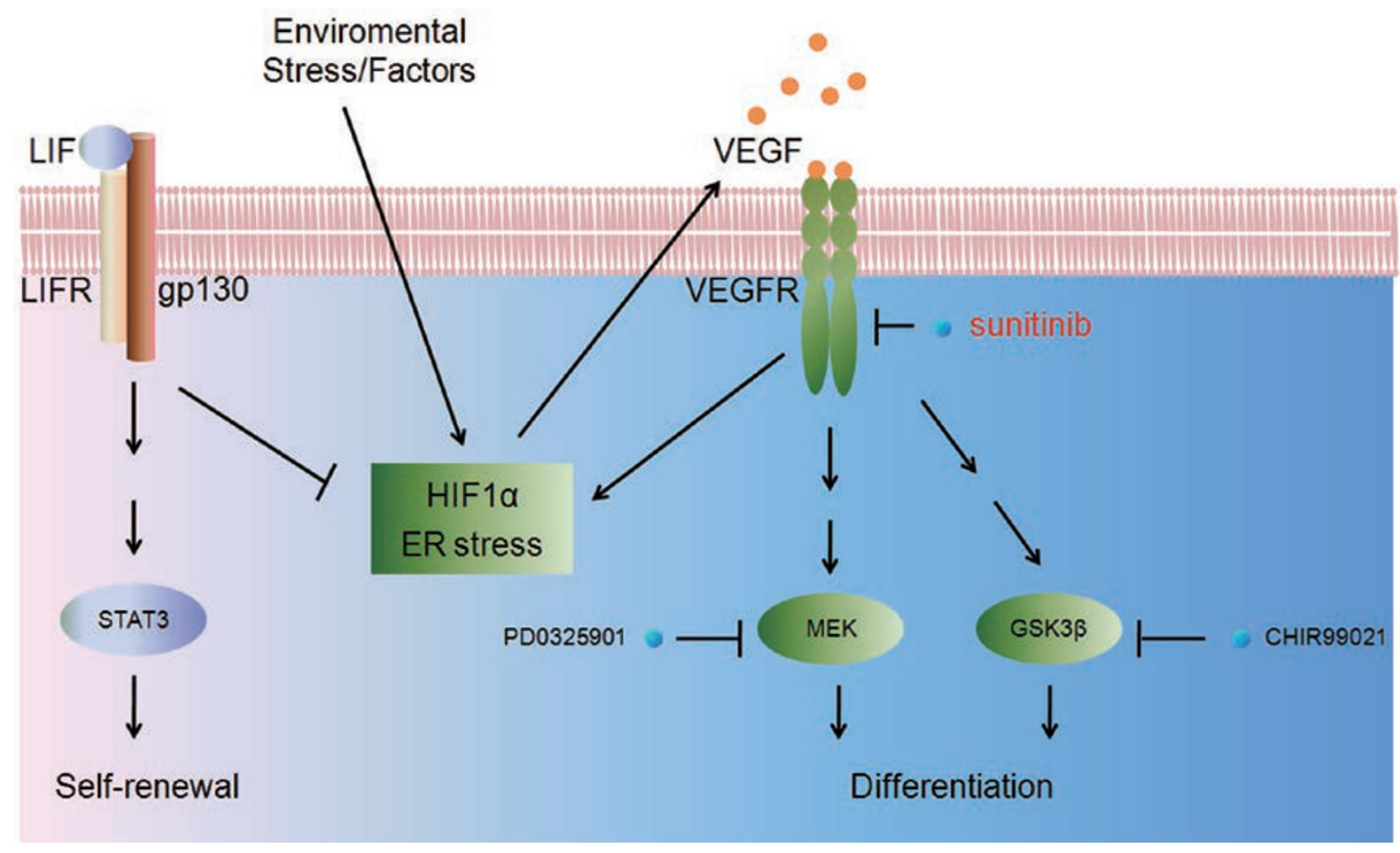

Figure 7 Schematic representation of the autocrine VEGF signaling in mESCs. Production of VEGF is activated via HIF1 $\alpha$ and/or ER stress, presumably due to environmental stress in the in vitro culture. VEGF activates VEGFRs expressed on the surface of the mESCs, inducing the activation of MEK, GSK3 $\beta$ and possibly other pathways that promote differentiation and lineage commitment of mESCs. Blocking VEGFRs with sunitinib inhibits both the MEK and GSK3 pathways and the feed-forward loop between HIF1 $\alpha /$ ER stress and VEGF/VEGFR, and thus prevents the spontaneous differentiation of mESCs.

early embryos. It is quite remarkable that the pluripotent cells in the ICM only exist transiently as they undergo programmed differentiation, while their pluripotency could be captured in vitro. The initial isolation and maintenance of mESCs relies on 'feeder cells' and serum supplementation, but later studies identified LIF and BMP4 as the key extracellular stimulants required for the sustained proliferation and self-renewal of mESCs [2, 37]. Similarly, hESCs can also be cultured in vitro, although they require the activation of fibroblast growth factor (FGF2) and TGFß/activin/Nodal pathways for maintenance $[38,39]$. In addition, modulation of the WNT pathway in mouse and human ESCs by inhibiting GSK3 $\beta$ activity, or adding WNT ligands, also enables short-term maintenance of the undifferentiated state [40].

Many of these growth factors exert their actions in ESCs in an autocrine manner. For example, FGF2 is believed to be secreted by hESCs to induce the expression of TGF $\beta$ family members such as Nodal, TGF $\beta$ and activin that activate the SMAD2/3 pathway which help maintain pluripotency [38]. In contrast, FGF4 is produced in $\mathrm{mESCs}$ as an autocrine factor that activates the ERK pathway and promotes exit from self-renewal into lineage commitment [4]. The latter leads to the dis- covery that the combination of inhibitors of ERK and GSK3 pathways (2i) are able to sustain the self-renewal of mESCs in the absence of extrinsic signals [5]. It has been suggested that mESCs cultured under these conditions are in a ground or 'naive' state and equivalent to cells from the early epiblasts. In this study, we identified VEGF as another autocrine factor that promotes lineage commitment of mESCs even in the presence of LIF. Blocking VEGF signaling with sunitinib, or by knocking down VEGFRs, not only maintained the mESCs in the undifferentiated state without the need for feeder cells or LIF, but also facilitated transcription factor-mediated pluripotency reprogramming in MEFs.

It is well accepted that VEGF produced by tumor cells acts on neighboring VEGFR-expressing endothelial cells to promote angiogenesis for continued tumor growth [41]. This concept has been challenged by reports demonstrating expression of the VEGFRs on tumor cells. An intriguing finding related to the autocrine function of VEGF signaling in tumors was that VEGF secreted by tumor cells triggers tumor cell proliferation by binding VEGFR1 expressed on the same cell [42]. Moreover, in patients with lung cancer, an increase in gene copy number of VEGFR2 is associated with chemoresistance 
and shorter survival [43]. Autocrine VEGF signaling has also been reported to play important roles in all aspects of breast tumor biology, including adhesion, survival, migration and invasion of the cancer cells [44]. In physiological condition, cell-autonomous VEGF signaling pathway has been demonstrated to be required for the homeostasis of blood vessels in the adult even though it is dispensable for the angiogenic cascade [45]. In ESC or iPSC cultures, the addition of exogenous VEGF has been demonstrated to induce endothelium and cardiac myocyte differentiation $[15,16]$, but the autocrine function of VEGFs has rarely been discussed. Our data demonstrated that the mESCs express both VEGF and VEGFRs, and the activation of this pathway leads to spontaneous differentiation. Interestingly, blocking the VEGF pathways with sunitinib decreased the expression of VEGFA (Figure $4 \mathrm{G}$ and $4 \mathrm{H}$ ) in mESCs, indicating the possible existence of an autocrine feed-forward loop which contributed to the continuous increase of VEGF secretion in long-term ESC culture. Such autocrine feed-forward loop of VEGF/ VEGFRs has also been demonstrated in lung cancer [46].

VEGFs are dramatically upregulated under hypoxic conditions. Hypoxia allows the stabilization of HIFs that bind to the promoter region of VEGFA [47]. Under different cultivation conditions, the role of hypoxia and HIFs in the fate determination of ESCs remains controversial. It has been reported that hypoxia prevents ESC differentiation and promotes iPSC induction [24]. Conversely, other report suggested that hypoxia or HIFs enhance the differentiation of hESC and mESC toward cardiomyocytes [48]. Exogenous expression of HIF-1 $\alpha$ also promotes cardiac differentiation of mESCs [49], while HIF-1 $\alpha$-knockout mESCs do not produce spontaneously beating cells [50]. Recently, VEGF, the important downstream protein of HIF $1 \alpha$, was proved to be indispensable in effective cardiac myocyte differentiation of human iPSCs [16]. In addition, hypoxic priming of mESCs accelerates vascular-lineage differentiation through HIF1-mediated VEGF expression and Oct4 suppression [27]. Our findings support the notion that the activation of HIF $1 \alpha$ and subsequent VEGF secretion from mESCs facilitated the meso-endoderm lineage commitment of these cells. Blocking HIF $1 \alpha$ reduced the expression of VEGF and promoted self-renewal of mESCs. Our data also demonstrated that ER stress plays a role in VEGF secretion in mESCs. It has been reported that hESCs must resolve ER stress related to culture conditions to survive and maintain pluripotency [32]. Blocking ER stress also reduced the expression of VEGF and promoted self-renewal of mESCs. Interestingly, we also demonstrated that LIF might also exert its self-renewal-promoting effect by inhibiting HIF $1 \alpha$ and ER stress pathways, thus blocking the activation of the autocrine VEGF signaling in $\mathrm{mESCs}$ (Figure 7).

Sunitinib (marketed as Sutent) is an orally available multi-targeted RTK inhibitor that was approved by the FDA for the treatment of renal cell carcinoma and imatinib-resistant gastrointestinal stromal tumor. Although mainly targeting VEGFRs and PDGFRs, it also inhibits other RTKs including KIT, FLT3, and RET [19]. In our study, we have demonstrated the expression of VEGF/ VEGFRs and PDGF/PDGFR in mESCs, and the activation of PDGF pathway might induce differentiation in a paracrine fashion, while the activation of VEGF pathway might induce differentiation in an autocrine way. Although we have demonstrated the critical role of VEGFR2 in spontaneous differentiation of mESCs by the knockdown and neutralizing antibody studies, sunitinib might exert its effect by blocking additional RTKs. For example, SCF and its receptor c-Kit play important roles in hematopoiesis, spermatogenesis and melanogenesis [51]. Mice that do not express SCF or c-Kit both die from severe anemia [51]. SCF may serve as guidance cues that direct hematopoietic stem cells to their stem cell niche. Flt3 is expressed on many hematopoietic progenitor cells, and play an important role in the development of haematopoietic stem cells and progenitor cells. Mutation in Flt3 gene is associated with acute myeloid leukemia [52]. CSF-1R, also known as macrophage CSF-1R, controls the differentiation and function of macrophages [53]. Mutations in CSF-1R are associated with chronic myelomonocytic leukemia and type M4 acute myeloblastic leukemia [54]. Although the expression and function of these RTKs have rarely been studied in mESCs, their activation all seem to lead to differentiation toward meso-endoderm lineages. Therefore, blocking any of these RTKs may also contribute to the self-renewal-promoting effect of sunitinib.

In conclusion, we have discovered the critical roles of autocrine VEGF signaling in the spontaneous differentiation of mESCs in in vitro culture. Blocking VEGF signaling with sunitinib, other RTK inhibitors targeting VEGFR, VEGFR neutralizing antibody, or by knocking down VEGFRs, was capable of maintaining the mESCs in the undifferentiated state without the need for feeder cells or LIF. Sunitinib could also be used to facilitate the derivation of mESCs from the blastocysts, and the mESCs maintained in sunitinib-containing medium remained pluripotent and were able to contribute to chimeric mouse. Sunitinib was also able to promote iPSC generation from MEFs. These results indicate the existence of an intrinsic differentiation pathway of mESCs by activating the VEGF signaling in an autocrine way. Blocking VEGF signaling with sunitinib or other small molecules 
help to maintain the mESCs in the ground state of pluripotency.

\section{Materials and Methods}

\section{ES cell culture}

E14 and OG2-ES cells were maintained feeder free on gelatin-coated plastics in mES media (DMEM with 15\% FBS (Hyclone 30070.03), 2 mM GlutaMAX, $0.1 \mathrm{mM}$ non-essential amino acids (NEAA), $0.1 \mathrm{mM} \beta$-mercaptoethanol, $100 \mathrm{U} / \mathrm{ml}$ penicillin and 100 $\mu \mathrm{g} / \mathrm{ml}$ streptomycin) supplemented with $1000 \mathrm{U} / \mathrm{ml}$ LIF and $2 \mathrm{i}$ ( $3 \mu \mathrm{M}$ CHIR99021 and $3 \mu \mathrm{M}$ PD0325901) and passaged every 3 days. In testing conditions, ES cells were cultured in mES media supplemented with LIF, $2 \mathrm{i}$, growth factors $\left(\mathrm{mVEGF}_{164}\right.$ and hPDGFBB) or other chemicals as indicated. In serum-free conditions, ES cells were cultured in N2B27 media (DMEM/F12 with 1/200 B27 supplement, 1/100 N2 supplement, 2 mM GlutaMAX, 0.1 mM NEAA and $0.1 \mathrm{mM} \beta$-mercaptoethanol) supplemented with LIF, 2i, growth factors or other chemicals as indicated.

\section{Derivation of ES cells with sunitinib}

Blastocysts from female ICR mice (Shanghai SLAC Lab Animal) were flushed from uterine horns with M2 medium (Sigma-Aldrich) 3.5 days post coitum. Isolated blastocysts were plated onto irradiated MEF feeder layer in mES medium containing sunitinib $(1 \mu \mathrm{M})$ and $2000 \mathrm{U} / \mathrm{ml}$ LIF. To obtain ES cell colonies, blastocyst outgrowths were disaggregated with trypsin and re-plated on gelatin-coated plastic wells with sunitinib and LIF. ESCs were expanded by passaging in $\mathrm{mES}$ medium supplemented with sunitinib $(1 \mu \mathrm{M})$. After six passages in sunitinib-containing mES medium, cells were injected into the $\mathrm{C} 57 \mathrm{BL} / 6$ blastocysts to test for chimera generation.

\section{Mouse iPSC generation}

OG2 mice, which carry a transgenic GFP gene driven by Oct4 promoter, were mated with C57BL/6J. MEFs were isolated from E13.5 embryos heterozygous for the Oct4-GFP transgenic allele. Gonads and internal organs were removed before MEF isolation. MEFs were grown in DMEM supplemented with $10 \%$ FBS, $2 \mathrm{mM}$ GlutaMAX, $0.1 \mathrm{mM}$ NEAA, $100 \mathrm{U} / \mathrm{ml}$ penicillin and $100 \mu \mathrm{g} / \mathrm{ml}$ streptomycin. MEFs in early passages (up to passage 3 ) were used for further experiments.

Retroviruses were produced by transfection of plat-E cells with pMXs retroviral vectors containing the coding sequences of mouse Oct4, Sox2, Klf4 and c-Myc (Addgene). MEFs were seeded at a density of 150000 cells/well into six-well plate $18 \mathrm{~h}$ before infection. Virus-containing supernatants, supplemented with $8 \mu \mathrm{g} / \mathrm{ml}$ polybrene, were added into the plates of MEF culture and centrifuged at $2500 \mathrm{rpm}$ for $90 \mathrm{~min}$. Medium was changed immediately after virus transduction and this day is counted as "day 0". Two days post virus infection, MEFs were reseeded at a density of 3 000 cells/well into 96-well plates pre-seeded with irradiated MEF feeder cells, supplemented with mES medium containing $1000 \mathrm{U} /$ ml LIF. On day 6, culture medium was replaced with KSR medium (knockout DMEM supplemented with 15\% KSR, 2 mM L-GlutaMAX, $0.1 \mathrm{mM}$ NEAA, $0.1 \mathrm{mM} \beta$-mercaptoethanol, $1000 \mathrm{U} / \mathrm{ml}$ LIF, $100 \mathrm{U} / \mathrm{ml}$ penicillin and $100 \mu \mathrm{g} / \mathrm{ml}$ streptomycin). Chemicals were added from day 3 with various durations. $\mathrm{GFP}^{+}$colonies were counted using a high-content screening instrument Acumen (TTP
Labtech) on day 14 post infection. Colonies were also trypsinized and analyzed using guava easyCyte Flow Cytometer (Millipore).

For reduced factor-induced iPSC, $150000 \mathrm{MEFs}$ cells $(2 \mathrm{~F}$, Oct4 and Klf4) or 200000 cells (1F, Oct4) per well were seeded in six-well plate $18 \mathrm{~h}$ before infection. Supernatants containing $2 \mathrm{~F}$ or $1 \mathrm{~F}$ were used for infection as above. Medium was changed immediately after virus transduction and this day is counted as "day 0". MEFs were infected twice, 2 days post virus infection, and MEFs were supplemented with KSR medium containing $10 \mathrm{ng} / \mathrm{ml}$ vitamin C, $1 / 100 \mathrm{~N} 2$ supplement and $10 \mathrm{ng} / \mathrm{ml}$ bFGF. Chemicals were added from day 3 to day 14 .

\section{Statistic analysis}

Values are reported as the mean \pm SEM. $P$-values were calculated by Student's $t$-test. All graphs are plotted with GraphPad Prism 5 software.

Detailed methods are described in the Supplementary information, Data S1.

\section{Acknowledgments}

This work was supported by grants from Chinese Academy of Sciences (XDA01040301), Ministry of Science and Technology of China (2009CB940900, 2011CB965104, 2011DFB30010, 2012ZX09301001-005) and Shanghai Commission of Science and Technology (12XD1402100).

\section{References}

1 Ulloa-Montoya F, Verfaillie CM, Hu WS. Culture systems for pluripotent stem cells. J Biosci Bioeng 2005; 100:12-27.

2 Williams RL, Hilton DJ, Pease S, et al. Myeloid leukaemia inhibitory factor maintains the developmental potential of embryonic stem cells. Nature 1988; 336:684-687.

3 Ying QL, Nichols J, Chambers I, Smith A. BMP induction of Id proteins suppresses differentiation and sustains embryonic stem cell self-renewal in collaboration with STAT3. Cell 2003; 115:281-292.

4 Kunath T, Saba-El-Leil MK, Almousailleakh M, Wray J, Meloche S, Smith A. FGF stimulation of the Erk1/2 signalling cascade triggers transition of pluripotent embryonic stem cells from self-renewal to lineage commitment. Development 2007; 134:2895-2902.

5 Ying QL, Wray J, Nichols J, et al. The ground state of embryonic stem cell self-renewal. Nature 2008; 453:519-523.

6 Zhang R, Zhang LH, Xie X. iPSCs and small molecules: a reciprocal effort towards better approaches for drug discovery. Acta Pharmacol Sin 2013; 34:765-776.

7 Dutta D, Ray S, Home P, Larson M, Wolfe MW, Paul S. Self-renewal versus lineage commitment of embryonic stem cells: protein kinase C signaling shifts the balance. Stem cells 2011; 29:618-628.

8 Olsson AK, Dimberg A, Kreuger J, Claesson-Welsh L. VEGF receptor signalling - in control of vascular function. Nat Rev Mol Cell Biol 2006; 7:359-371.

9 Ng YS, Rohan R, Sunday ME, Demello DE, D'Amore PA. Differential expression of VEGF isoforms in mouse during development and in the adult. Dev Dyn 2001; 220:112-121. 
10 Carmeliet P, Ferreira V, Breier G, et al. Abnormal blood vessel development and lethality in embryos lacking a single VEGF allele. Nature 1996; 380:435-439.

11 Shalaby F, Rossant J, Yamaguchi TP, et al. Failure of blood-island formation and vasculogenesis in Flk-1-deficient mice. Nature 1995; 376:62-66.

12 Miquerol L, Langille BL, Nagy A. Embryonic development is disrupted by modest increases in vascular endothelial growth factor gene expression. Development 2000; 127:3941-3946.

13 Gerber HP, Malik AK, Solar GP, et al. VEGF regulates haematopoietic stem cell survival by an internal autocrine loop mechanism. Nature 2002; 417:954-958.

14 Takahashi H, Shibuya M. The vascular endothelial growth factor (VEGF)/VEGF receptor system and its role under physiological and pathological conditions. Clin Sci 2005; 109:227241.

15 Zeng L, Xiao Q, Margariti A, et al. HDAC3 is crucial in shear- and VEGF-induced stem cell differentiation toward endothelial cells. J Cell Biol 2006; 174:1059-1069.

16 Ye L, Zhang S, Greder L, et al. Effective cardiac myocyte differentiation of human induced pluripotent stem cells requires VEGF. PLoS One 2013; 8:e53764.

17 Chung AS, Ferrara N. Developmental and pathological angiogenesis. Annu Rev Cell Dev Biol 2011; 27:563-584.

18 Sato N, Meijer L, Skaltsounis L, Greengard P, Brivanlou AH. Maintenance of pluripotency in human and mouse embryonic stem cells through activation of Wnt signaling by a pharmacological GSK-3-specific inhibitor. Nat Med 2004; 10:55-63.

19 Roskoski R Jr. Sunitinib: a VEGF and PDGF receptor protein kinase and angiogenesis inhibitor. Biochem Biophys Res Commun 2007; 356:323-328.

20 Olsson AK, Dimberg A, Kreuger J, Claesson-Welsh L. VEGF receptor signalling - in control of vascular function. Nat Rev Mol Cell Biol 2006; 7:359-371.

21 Kou R, SenBanerjee S, Jain MK, Michel T. Differential regulation of vascular endothelial growth factor receptors (VEGFR) revealed by RNA interference: interactions of VEGFR-1 and VEGFR-2 in endothelial cell signaling. Biochemistry 2005; 44:15064-15073.

22 Suchting S, Freitas C, le Noble F, et al. The Notch ligand Delta-like 4 negatively regulates endothelial tip cell formation and vessel branching. Proc Natl Acad Sci USA 2007; 104:3225-3230.

23 Wilson WR, Hay MP. Targeting hypoxia in cancer therapy. Nat Rev Cancer 2011; 11:393-410.

24 Ezashi T, Das P, Roberts RM. Low $\mathrm{O}_{2}$ tensions and the prevention of differentiation of hES cells. Proc Natl Acad Sci USA 2005; 102:4783-4788.

25 Yoshida Y, Takahashi K, Okita K, Ichisaka T, Yamanaka S. Hypoxia enhances the generation of induced pluripotent stem cells. Cell Stem Cell 2009; 5:237-241.

26 Han Y, Kuang SZ, Gomer A, Ramirez-Bergeron DL. Hypoxia influences the vascular expansion and differentiation of embryonic stem cell cultures through the temporal expression of vascular endothelial growth factor receptors in an ARNT-dependent manner. Stem Cells 2010; 28:799-809.

27 Lee SW, Jeong HK, Lee JY, et al. Hypoxic priming of mESCs accelerates vascular-lineage differentiation through HIF1-mediated inverse regulation of Oct4 and VEGF. EMBO Mol Med
2012; 4:924-938.

28 Piret JP, Mottet D, Raes M, Michiels C. $\mathrm{CoCl}_{2}$, a chemical inducer of hypoxia-inducible factor-1, and hypoxia reduce apoptotic cell death in hepatoma cell line HepG2. Ann NY Acad Sci 2002; 973:443-447.

29 Theriault JR, Felts AS, Bates BS, et al. Discovery of a new molecular probe ML228: an activator of the hypoxia inducible factor (HIF) pathway. Bioorg Med Chem Lett 2012; 22:76-81.

30 Narita T, Yin S, Gelin CF, et al. Identification of a novel small molecule HIF-1alpha translation inhibitor. Clin Cancer Res 2009; 15:6128-6136.

31 Ghosh R, Lipson KL, Sargent KE, et al. Transcriptional regulation of VEGF-A by the unfolded protein response pathway. PLoS One 2010; 5:e9575.

32 Blanco-Gelaz MA, Suarez-Alvarez B, Ligero G, et al. Endoplasmic reticulum stress signals in defined human embryonic stem cell lines and culture conditions. Stem Cell Rev 2010; 6:462-472.

33 Liu L, Liu C, Zhong Y, Apostolou A, Fang S. ER stress response during the differentiation of $\mathrm{H} 9$ cells induced by retinoic acid. Biochem Biophys Res Commun 2012; 417:738-743.

34 Ozcan L, Tabas I. Role of endoplasmic reticulum stress in metabolic disease and other disorders. Ann Rev Med 2012; 63:317-328.

35 Kubota Y, Hirashima M, Kishi K, Stewart CL, Suda T. Leukemia inhibitory factor regulates microvessel density by modulating oxygen-dependent VEGF expression in mice. $J$ Clin Invest 2008; 118:2393-2403.

36 Wang Q, Xu X, Li J, et al. Lithium, an anti-psychotic drug, greatly enhances the generation of induced pluripotent stem cells. Cell Res 2011; 21:1424-1435.

37 Smith AG, Heath JK, Donaldson DD, et al. Inhibition of pluripotential embryonic stem cell differentiation by purified polypeptides. Nature 1988; 336:688-690.

38 Vallier L, Alexander M, Pedersen RA. Activin/Nodal and FGF pathways cooperate to maintain pluripotency of human embryonic stem cells. J Cell Sci 2005; 118:4495-4509.

39 Dvorak P, Dvorakova D, Koskova S, et al. Expression and potential role of fibroblast growth factor 2 and its receptors in human embryonic stem cells. Stem Cells 2005; 23:1200-1211.

40 Sato N, Meijer L, Skaltsounis L, Greengard P, Brivanlou AH. Maintenance of pluripotency in human and mouse embryonic stem cells through activation of Wnt signaling by a pharmacological GSK-3-specific inhibitor. Nat Med 2004; 10:55-63.

41 Ferrara N, Gerber HP, LeCouter J. The biology of VEGF and its receptors. Nat Med 2003; 9:669-676.

42 Lichtenberger BM, Tan PK, Niederleithner H, Ferrara N, Petzelbauer P, Sibilia M. Autocrine VEGF signaling synergizes with EGFR in tumor cells to promote epithelial cancer development. Cell 2010; 140:268-279.

43 Yang F, Tang XM, Riquelme E, et al. Increased VEGFR-2 gene copy is associated with chemoresistance and shorter survival in patients with non-small-cell lung carcinoma who receive adjuvant chemotherapy. Cancer Res 2011; 71:55125521.

44 Perrot-Applanat M, Di Benedetto M. Autocrine functions of VEGF in breast tumor cells: adhesion, survival, migration and invasion. Cell Adh Migr 2012; 6:547-553.

45 Lee S, Chen TT, Barber CL, et al. Autocrine VEGF signaling 
is required for vascular homeostasis. Cell 2007; 130:691-703.

46 Chatterjee S, Heukamp LC, Sioba M, et al. Tumor VEGF:VEGFR2 autocrine feed-forward loop triggers angiogenesis in lung cancer. J Clin Invest 2013; 123:1732-1740.

47 Pugh CW, Ratcliffe PJ. Regulation of angiogenesis by hypoxia: role of the HIF system. Nat Med 2003; 9:677-684.

48 Niebruegge S, Bauwens CL, Peerani R, et al. Generation of human embryonic stem cell-derived mesoderm and cardiac cells using size-specified aggregates in an oxygen-controlled bioreactor. Biotechnol Bioeng 2009; 102:493-507.

$49 \mathrm{Ng} \mathrm{KM}$, Lee YK, Chan YC, et al. Exogenous expression of HIF-1 alpha promotes cardiac differentiation of embryonic stem cells. J Mol Cell Cardiol 2010; 48:1129-1137.

50 Ateghang B, Wartenberg M, Gassmann M, Sauer H. Regulation of cardiotrophin-1 expression in mouse embryonic stem cells by HIF-1alpha and intracellular reactive oxygen species. J Cell Sci 2006; 119:1043-1052.

51 Lennartsson J, Ronnstrand L. Stem cell factor receptor/c-Kit: from basic science to clinical implications. Physiol Rev 2012; 92:1619-1649.
52 Masson K, Ronnstrand L. Oncogenic signaling from the hematopoietic growth factor receptors c-Kit and Flt3. Cell Signal 2009; 21:1717-1726.

53 Hume DA, MacDonald KPA. Therapeutic applications of macrophage colony-stimulating factor-1 (CSF-1) and antagonists of CSF-1 receptor (CSF-1R) signaling. Blood 2012; 119:1810-1820.

54 Ridge SA, Worwood M, Oscier D, Jacobs A, Padua RA. FMS mutations in myelodysplastic, leukemic, and normal subjects. Proc Natl Acad Sci USA 1990; 87:1377-1380.

(Supplementary information is linked to the online version of the paper on the Cell Research website.)

(c) (i) (s) $\odot$ This work is licensed under the Creative Commons Attribution-NonCommercial-No Derivative Works 3.0 Unported License. To view a copy of this license, visit http:// creativecommons.org/licenses/by-nc-nd/3.0 